\title{
SIRT2 mediates multidrug resistance in acute myelogenous leukemia cells via ERK1/2 signaling pathway
}

\author{
HUA XU, YUANYE LI, LONG CHEN, CHIJUAN WANG, QI WANG, \\ HAIRUI ZHANG, YANI LIN, QINGHUA LI and TIANXIANG PANG
}

\begin{abstract}
State Key Laboratory of Experimental Hematology, Institute of Hematology and Blood Diseases Hospital, Chinese Academy of Medical Sciences and Peking Union Medical College, Tianjin 300020, P.R. China
\end{abstract}

Received July 25, 2015; Accepted September 4, 2015

DOI: $10.3892 /$ ijo.2015.3275

\begin{abstract}
SIRT2, one of nicotinamide adenine dinucleotide $\left(\mathrm{NAD}^{+}\right)$-dependent class III histone deacetylase family proteins, has been found to be involved in the proliferation and survival of acute myeloid leukemia (AML) cells. However, its effect on drug resistance on chemoresistant AML cells is unclear. In the present study, we first found that SIRT2 was expressed at higher level in the relapsed AML patients than the newly diagnosed patients. Consistent with this observation, the expression level of SIRT2 was higher in HL60/A cells than that in HL60 cells. Depletion of SIRT2 by shRNAs in HL60/A cells resulted in decreased MRP1 level, enhanced drug accumulation and triggered more apoptosis. By contrast, overexpression of SIRT2 in HL60 cells led to increased MRP1 level, drug efflux and attenuated drug sensitivity. Moreover, the decreased expression of phosphorylated ERK1/2 was detected in SIRT2-depleted HL60/A cells and increased expression of phosphorylated ERK1/2 was observed in SIRT2 overexpressed HL60 cells. Furthermore, blockage of ERK1/2 signaling pathway with the chemical inhibitor PD98059, further induced apoptosis of HL60/A cells conferred by SIRT2 depletion. Importantly, ERK1/2 inhibition was able to reverse the drug resistance of HL60 conferred by SIRT2 overexpression. Thus, our findings collectively suggested that the expression level of SIRT2 has a positive relationship with DNR/Ara-C resistance and activity of ERK1/2 signaling pathway. SIRT2 might regulate DNR/Ara-C sensitivity in AML cells at least partially through the ERK1/2 pathway.
\end{abstract}

Correspondence to: Professor Tianxiang Pang, State Key Laboratory of Experimental Hematology, Institute of Hematology and Blood Diseases Hospital, Chinese Academy of Medical Sciences and Peking Union Medical College, Nanjing Road 288, Tianjin 300020, P.R. China E-mail: pang@ihcams.ac.cn

Key words: SIRT2, acute myeloid leukemia, multidrug resistance, MRP1, ERK1/2

\section{Introduction}

Acute myeloid leukemia (AML) is a genetically heterogeneous disorder, characterized by uncontrolled clonal proliferation of immature myeloid cells in the bone marrow and blood with concurrent depletion of effective hematopoiesis $(1,2)$. The standard treatment of AML with cytotoxic chemotherapy has remained mostly unchanged over the past few decades, with dismal clinical outcome (3). One of the key issues of this poor result is the development of resistance to chemotherapeutic agents, which can lead to clinical relapse (4). Hence, novel antileukemia agents, especially for relapsed leukemias resistant to existing chemotherapeutic drugs are needed.

Multidrug resistance (MDR) is a major problem in the treatment of various human hematological malignancies. Overexpression of the drug transporter P-glycoprotein (P-gp) and/or multidrug resistance-associated protein (MRP1) has generally been reported to correlate with prognosis in AML $(5,6)$. MRP1, like P-gp belongs to the ATP binding cassette (ABC) superfamily of membrane transport protein (6). The difference between them is that the $190-\mathrm{kDa}$ MRP1 can be localized on both the plasma and intracytoplasmic membranes, which can cause intracellular or cytoplasmic sequestration of the drug (5). MRP1 overexpression leads to the failure of numerous chemotherapy protocols when there is without $\mathrm{P}-\mathrm{gp}$ overexpression, such as in HL60/A cells (7).

Sirtuins (SIRT1-7), the mammalian homologues of the silent information regulator 2 (Sir2) in yeast, are members of nicotinamide adenine dinucleotide $\left(\mathrm{NAD}^{+}\right)$-dependent class III histone deacetylase family proteins (8-10). Sirtuins have a critical role in genome stability, longevity, metabolism and aging $(9,10)$. Among them, SIRT1 is the direct homologue of the yeast Sir2 and has a wide range of cellular functions (11). Previous studies have suggested that SIRT1 has oncogenic ability in hematological malignancies, such as CLL, AML and even can be seen as a therapeutic target for CML and AML treatment to overcome resistance (12-14). Little is known about SIRT2, which is the primary cytoplasmic Sirtuin but shuttles continuously between the cytoplasmic and nuclear compartments during interphase and thus, it has been reported to participate in cell cycle progression, cell differentiation, and oxidative stress by modifing other proteins and regulating gene expression $(15-17,24,29)$. SIRT2 has been 
Table I. Characteristics of the patients.

\begin{tabular}{|c|c|c|c|c|c|c|c|c|}
\hline & Normal & Children-ALL & ALL & ET & CML & PV & MPN & AML \\
\hline Number of patients & 6 & 25 & 30 & 10 & 11 & 8 & 11 & 25 \\
\hline \multicolumn{9}{|l|}{ Age (years) } \\
\hline Median & 28 & 6 & 34 & 40 & 39 & 40 & 32 & 37 \\
\hline Range & $9-36$ & $2-13$ & $19-53$ & $25-55$ & $5-79$ & $27-54$ & $28-50$ & $1-60$ \\
\hline \multicolumn{9}{|l|}{ Gender } \\
\hline Female & 2 & 11 & 9 & 3 & 4 & 4 & 5 & 11 \\
\hline Male & 4 & 14 & 21 & 7 & 7 & 4 & 6 & 14 \\
\hline
\end{tabular}

ALL, acute lymphoblastic leukemia; ET, essential thrombocythemia; CML, chronic myeloid leukemia; PV, polycythemia vera; MPN; myeloproliferative neoplasm; AML, acute myeloid leukemia.

suggested to participate in glioma, breast, lung cancer, hepatocellular carcinoma, though, there are few studies in leukemia $(8,18-20)$.

In the present study, we found that SIRT2 was overexpressed in the relapsed patients with AML compared to the newly diagnosed, and both groups were increased from the normal levels, and the MRP1-mediated multidrug resistance AML cell line HL60/A could be reversed by inhibition of SIRT2.

\section{Materials and methods}

Patients and cell culture. Following informed consent, we collected bone marrow samples of patients in the Hospital of Blood Diseases. The patients included 25 children with acute lymphoblastic leukemia (children-ALL), 30 acute lymphoblastic leukemia (ALL), 10 essential thrombocythemia (ET), 11 chronic myeloid leukemia (CML), 8 polycythemia vera (PV), 11 myeloproliferative neoplasm (MPN), 25 acute myeloid leukemia (AML) and 6 healthy volunteers (Table I). All the patients were newly diagnosed, except the 10 relapsed AML patients. Inclusion criteria for the present study were based on the European Leukemia Net (ELN) criteria. All the patient samples were treated in accordance with the Helsinki Declaration.

HL60 cells were preserved by our laboratory and its MRP-mediated multidrug resistance HL60/A cells were provided by the Pharmacology Laboratory at Institute of Hematology, Chinese Academy of Medical Sciences. They were cultured in RPMI-1640 (Gibco-BRL Life Technologies, Inc., Burlington, ON, Canada) containing $10 \%$ fetal bovine serum (FBS; HyClone Laboratories, Logan, UT, USA), $100 \mathrm{U} / \mathrm{ml}$ penicillin and $100 \mu \mathrm{g} / \mathrm{ml}$ streptomycin at $37^{\circ} \mathrm{C}$ in a humid atmosphere with $5 \% \mathrm{CO}_{2}$. HL60/A cells were grown in the presence of $1 \mu \mathrm{g} / \mathrm{ml}$ doxorubicin (DOX) to maintain resistance activity, which was removed 1 week before each assay.

Quantitative real-time PCR and western blot analysis. Total RNA was extracted using TRIzol reagent (Invitrogen, Grand Island, NY, USA) and converted to cDNA by using the SuperScript II RT (Invitrogen). Premier software 5 was used to design the primers for real-time quantitative PCR. Human GAPDH primers used as an internal control were
5'-GAAGGTGAAGGTCGGAGTC-3' (forward) and 5'-GAA GATGGTGATGGGATTTC-3' (reverse). Human SIRT2 primers were 5'-ACGCTGTCGCAGAGTCAT-3' (forward) and 5'-CGCTCCAGG GTATCTATGTT-3' (reverse). Realtime quantitative PCR was performed with SYBR-Green PCR kit (Takara, Shiga, Japan) on the ABI PRISM 7500 Sequence detection system. Thermal cycling conditions were $95^{\circ} \mathrm{C}$ for $30 \mathrm{sec}$, followed by 40 cycles of $5 \mathrm{sec}$ at $95^{\circ} \mathrm{C}$, and $34 \mathrm{sec}$ at $60^{\circ} \mathrm{C}$. PCR reactions were performed in a total volume of $20 \mu \mathrm{l}$, containing $2 \mu \mathrm{l}$ of sample cDNA, $0.2 \mu \mathrm{M}$ of each primer and the SYBR-Green PCR kit following the manufacturer's instructions. The expression level of SIRT2 was analyzed by the RQ value calculated through $2^{-\Delta \Delta \mathrm{Ct}}$ method $\left[\Delta \Delta \mathrm{Ct}-\left(\mathrm{Ct}_{\mathrm{SIRT} 2}\right.\right.$ - $\left.\left.\mathrm{Ct}_{\mathrm{GAPDH}}\right)_{\text {sample }}-\left(\mathrm{Ct}_{\mathrm{SIRT} 2}-\mathrm{Ct}_{\mathrm{GAPDH}}\right)_{\text {calibrator }}\right]$. Data are the means $\pm \mathrm{SD}$ of at least 3 independent experiments performed in triplicate.

Total protein was extracted using RIPA lysis buffer with $1 \mathrm{mM}$ PMSF (Sigma, St. Louis, MO, USA) and other protease inhibitors. Protein concentration was determined by the BCA assay (Beijing Solarbio Science and Technology Co., Ltd., Beijing, China) according to the manufacturer's instructions. The total cell lysates were heat-denatured at $100^{\circ} \mathrm{C}$ for 10 min before being run on $8-12 \%$ gradient SDS-PAGE. After SDS-PAGE, the proteins were transferred onto polyvinylidene difluoride membranes (Millipore, Bedford, MA, USA). Blots were incubated with the indicated antibodies, incubated in secondary antibody and then developed by using enhanced chemiluminescence detection reagent (GE Healthcare, Buckinghamshire, UK) and determined by densitometric analysis with a Lynx video densitometer (Biological Vision Inc., San Mateo, CA, USA). For western blot analysis, we purchased antibodies against GAPDH (sc-365062), SIRT2 (sc-20966), multidrug-resistance protein 1 (MRP1) (sc-7773), antiphosphospecfic ERK1/2 (sc-101760) and non-phosphorylated ERK1/2 (sc-135900) from Santa Cruz Biotechnology (Santa Cruz, CA, USA); p53 (9282), BCL-2 (2872) and Apoptosis Antibody Sampler kit (9915) from Cell Signaling Technology (Danvers, MA, USA).

Lentiviral shRNA vectors and transduction. Two different SIRT2-specific targeting sequences were designed with software from Ambion. The hairpins were synthesized and cloned into the lentiviral expression vector. The two shRNA vectors 
A

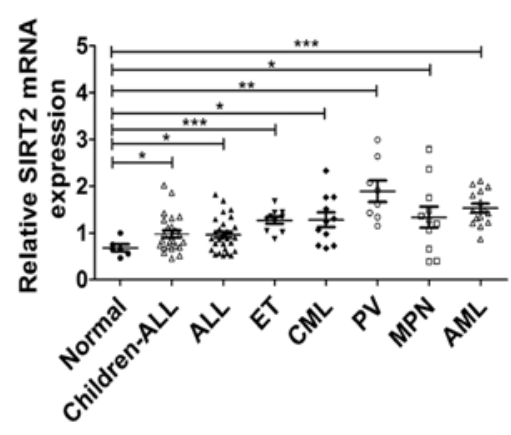

B

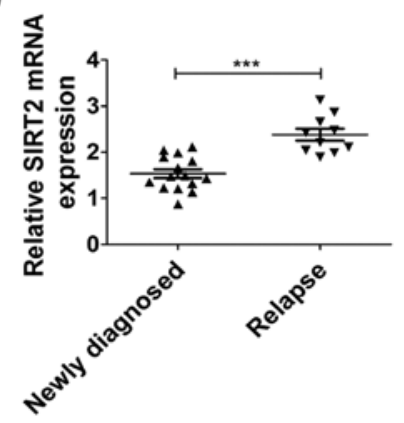

C

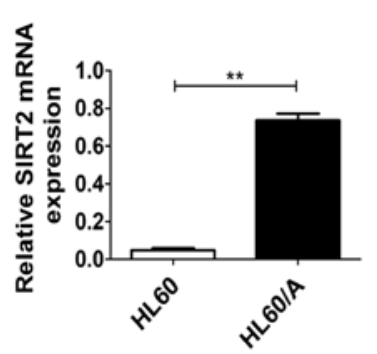

D

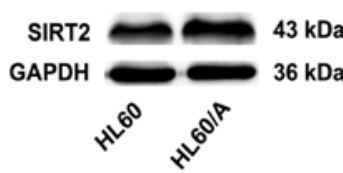

Figure 1. Expression of SIRT2 in patient samples and leukemic cells. (A) Real-time quantitative PCR was performed on templates of cDNA from different patient cells to detected SIRT2 mRNA expression level. (B) Expression patterns of SIRT2 in newly diagnosed and relapsed acute myelogenous leukemia patient samples were analyzed by real-time quantitative PCR. (C and D) The expression of SIRT2 in HL60 and HL60/A cells was detected by real-time quantitative PCR analysis on the mRNA expression level and western blot analysis on the protein expression level. GAPDH was used as an internal control for both real-time quantitative PCR and western blot analysis. Data are representative of at least three independent experiments. ${ }^{*} \mathrm{P}<0.05,{ }^{* *} \mathrm{P}<0.01,{ }^{* * *} \mathrm{P}<0.001$, compared with the control.

and empty vector were transfected respectively with packaging vectors into $293 \mathrm{~T}$ producer cells using Lipofectamine ${ }^{\mathrm{TM}}$ 2000 (Invitrogen) according to the protocol provided by the company. The supernatants were harvested after culturing for $48 \mathrm{~h}$ and concentrated by ultracentrifugation. Packaged SIRT2-shRNAs and the control vector were transfected into HL60/A cells separately. The transfectants were selected with puromycin and screened under a fluorescence microscope to observe GFP expression. SIRT2 knockdown efficiency was measured by real-time PCR and western blot analysis.

Lentiviral vector construction for SIRT2 overexpression and transfection. The complete coding region of SIRT2 gene was amplified by PCR and cloned into the lentiviral expression vector pCDH-CMV-MCS-EF1-GreenPuro-CD512B-1. The transfection protocol is described above.

Immunofluorescence assay. Cells with indicated treatments were fixed with $4 \%$ paraformaldehyde for $30 \mathrm{~min}$ and permeabilized with $0.5 \%$ Triton for 15 min. Cells were washed with ice-cold PBS, blocked with $0.5 \%$ BSA in PBS for $30 \mathrm{~min}$ and then incubated with a rabbit polyclonal antibody against SIRT2, followed by TRITC-conjugated goat anti-rabbit IgG antibody and stained nuclei with DAPI. The images were then visualized with Leica TCS SP2 confocal laser microscope (Perkin-Elmer, Waltham, MA, USA).

Measurement of intracellular doxorubicin. HL60/A, HL60 cells and their transfectants were treated with DOX $10 \mu \mathrm{mol} / 1$ in serum-free RPMI-1640 for $1 \mathrm{~h}$ and washed twice by PBS before imaging on Leica TCS SP2 confocal LAS for $1 \mathrm{~h}$ before imaging on Leica TCS SP 2 confocal laser microscope (Perkin-
Elmer). In another group, cells were incubated for $1.5 \mathrm{~h}$ in serum-free RPMI-1640 containing doxorubicin $10 \mu \mathrm{mol} / \mathrm{l}$ and analyzed by flow cytometry.

Cytotoxicity assay and analysis of apoptosis. Cells were seeded into 96-well culture plates at a density of $1 \times 10^{5}$ cells $/ \mathrm{ml}$ and serial concentration of daunorubicin (DNR) and arabinocytidine (Ara-C) were, respectively, added in a final volume of $100 \mu \mathrm{l} /$ well for $48 \mathrm{~h}$. The cytotoxic effects of drugs were determined by VersaMax microplate reader (Molecular Devices, Sunnyvale, CA, USA) at $570 \mathrm{~nm}$.

The apoptosis of the cells with or without DNR and Ara-C were measured with Annexin V-APC/PI apoptosis analysis kit (Tianjin Sungene Biotech Co., Ltd., Tianjin, China) as recommended by the manufacturer and analyzed by flow cytometry.

Statistical analysis. Each experiment was repeated at least three times. The data were summarized and presented as mean \pm SD. The statistical analyses were performed with Student's t-test using GraphPad Prism software (GraphPad Prism, San Diego, CA, USA). $\mathrm{P}<0.05$ was considered as statistically significant.

\section{Results}

Expression of SIRT2 in leukemia. We first compared SIRT2 expression of patients with different leukemias by real-time PCR. The real-time PCR analysis revealed that the expressions of SIRT2 in leukemia patients was higher than that in healthy subjects, although the degree of discrepancy varied (Fig. 1A). Then, we detected the expressions of SIRT2 in 10 relapsed AML patients and 15 newly diagnosed AML patients 
A

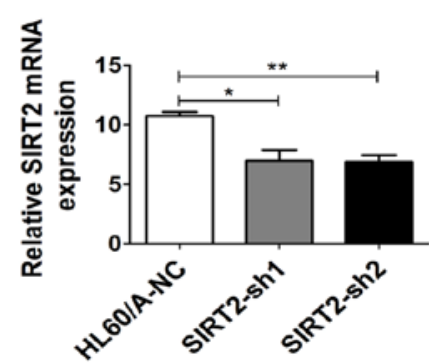

C

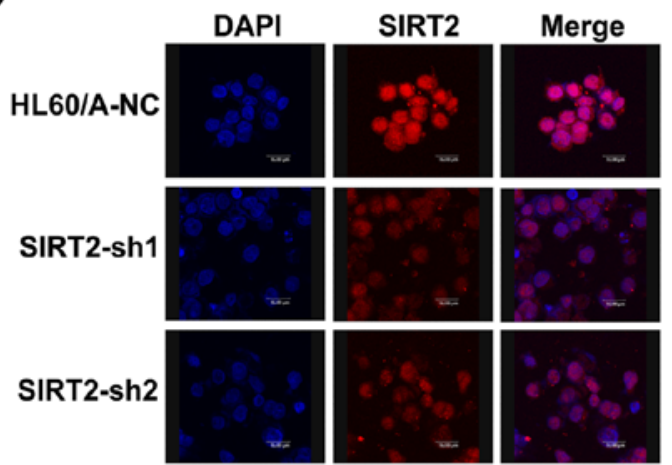

E

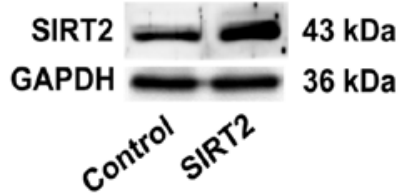

B

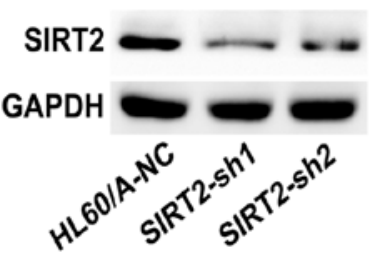

$43 \mathrm{kDa}$

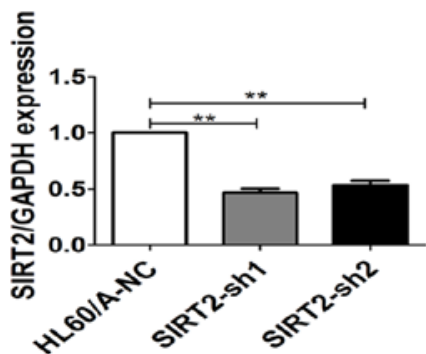

$36 \mathrm{kDa}$

D

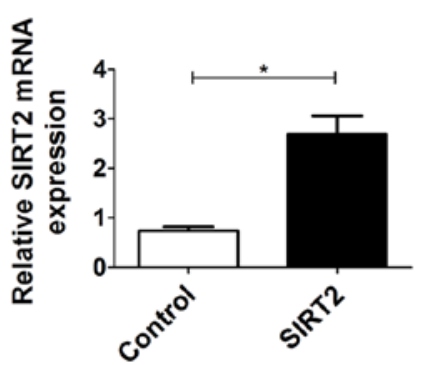

F

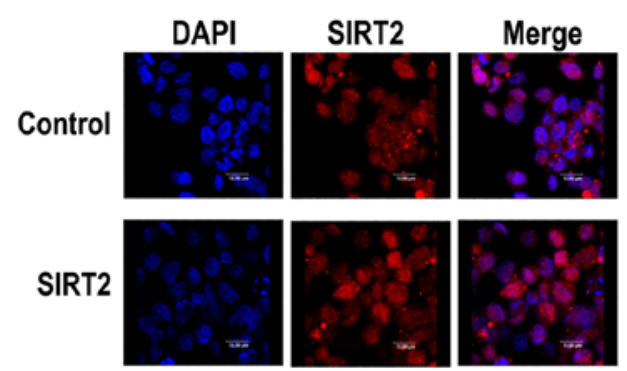

Figure 2. Validation of SIRT2 knockdown in HL60/A cells and SIRT2 overexpression in HL60 cells. (A) Relative SIRT2 mRNA expression was assessed in HL60/A cells transfected with SIRT2 short hairpin RNA plasmids by real-time quantitative PCR. (B) Lysates of HL60/A cells transfected with indicated plasmids were analyzed for SIRT2 protein by western blotting. The shRNA1 and shRNA2 were confirmed capable of inhibiting SIRT2 significantly (P<0.01). (C) Immunofluorescence assay was performed to detect the inhibition of SIRT2 observed by confocal laser microscope. Blue indicates DAPI and red SIRT2 staining. (D) Relative SIRT2 mRNA expression was assessed in HL60 cells transfected with SIRT2 overexpression plasmids by real-time quantitative PCR. (E) Lysates of HL60 cells transfected with indicated plasmids were analyzed for SIRT2 protein by western blotting. The result confirmed significant SIRT2 overexpression $(\mathrm{P}<0.05)$. (F) Immunofluorescence assay was performed to detect the overexpression of SIRT2 observed by a confocal laser microscope. Blue indicates DAPI and red SIRT2 staining. Each value indicated the mean \pm SD of triplicate in an independent experiment. ${ }^{*} \mathrm{P}<0.05$, ${ }^{* *} \mathrm{P}<0.01$, compared with control.

and the result showed that the expressions of SIRT2 in the relapsed AML patients were higher than that in the newly diagnosed patients (Fig. 1B). In addition, we also detected the expression of SIRT2 in HL60 acute myeloid leukemia cell lines and its MRP-mediated multidrug resistance HL60/A cells through real-time PCR and western blotting. Compared with SIRT2 expression in HL60 cells, SIRT2 level in HL60/A was higher (Fig. 1C and D). Both on the level of mRNA and protein the results showed that the expression of SIRT2 in HL60 cells and HL60/A cells were similar to our previous findings. These data not only indicate that the expression of SIRT2 in leukemia patients is higher than that in healthy volunteers, but also suggest that the overexpression of SIRT2 may associate with multidrug resistance in AML.

SIRT2 knockdown decreased the MRP1 level, enhanced drug accumulation and sensitivity to DNR and Ara-C. Two different SIRT2 specific shRNA plasmids were constructed and transfected into HL60/A cells, which had a relatively higher expression level of SIRT2. As expected, stable transfection experiments showed both shRNA plasmids could significantly inhibit SIRT2 expression in HL60/A cells in mRNA and protein levels (Fig. 2A and B). As both cytoplasmic and nuclear SIRT2 exist, the expression of SIRT2 in the HL60/A cells after transfection was detected by a confocal laser microscope. The results showed that the SIRT2 expression was significantly decreased, similarly to the results of western blotting (Fig. 2C).

We detected the MRP1 protein, which is known as a multidrug efflux pumps, and we found the MRP1 expression decreased after inhibition of SIRT2 in HL60/A cells (Fig. 3A).

After downregulation the expression of SIRT2, in HL60/A cells showed increased uptake of DOX compared with the control group HL60/A-NC cells (Fig. 3B). Flow cytometry was also used to measure the intracellular fluorescent of DOX, and similar result was obtained (Fig. 3C). 
A
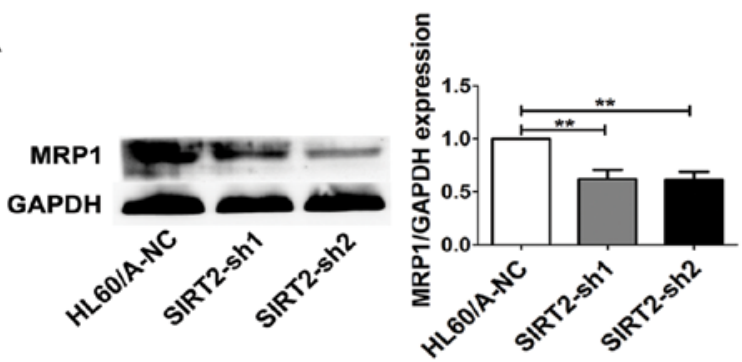

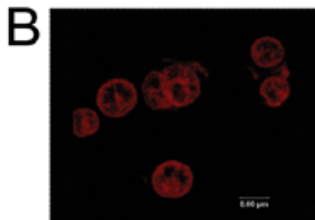

HL60/A-NC

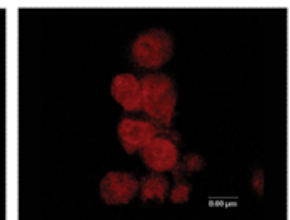

SIRT2-sh1

F

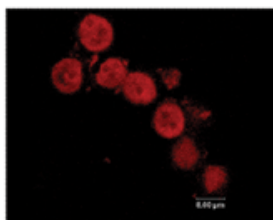

Control

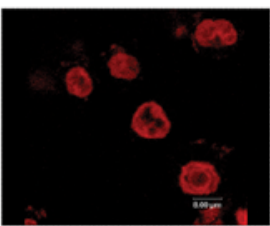

SIRT2

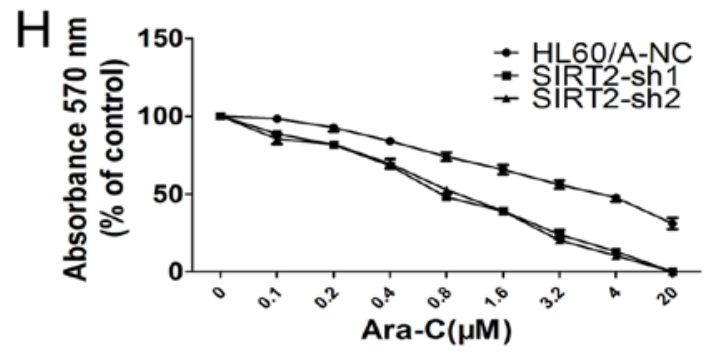

C

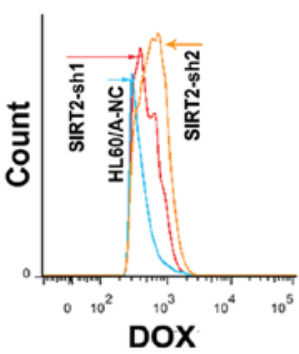

SIRT2-sh2
E

MRP1

GAPDH

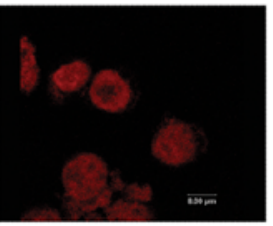

GAPDH

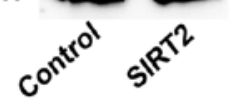

G
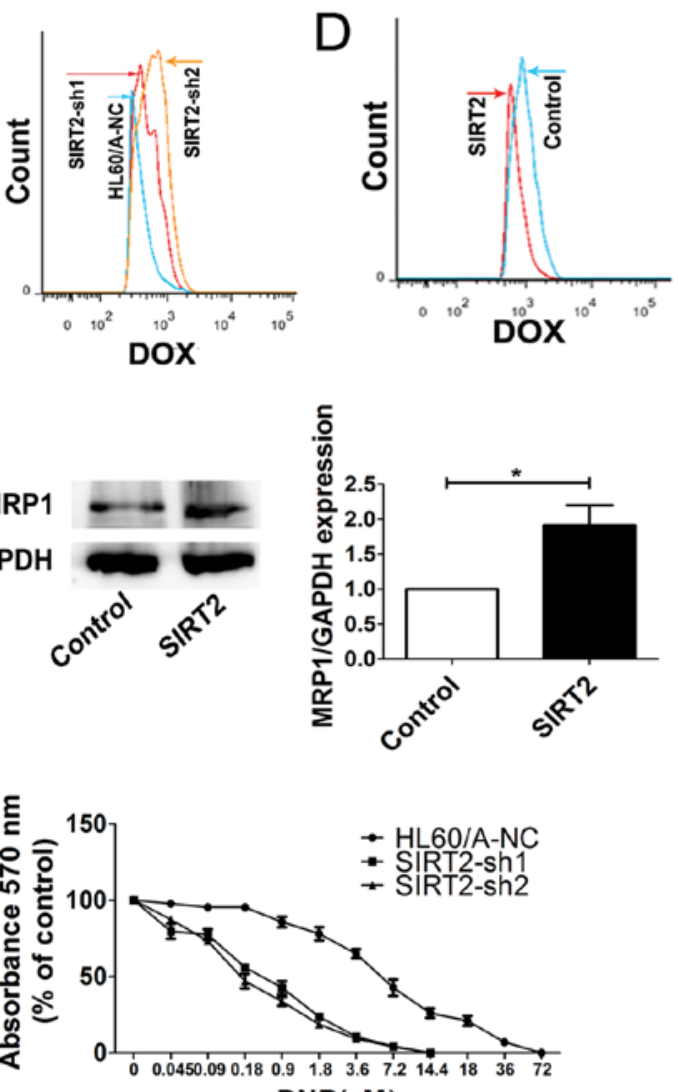

$\operatorname{DNR}(\mu \mathrm{M})$

I
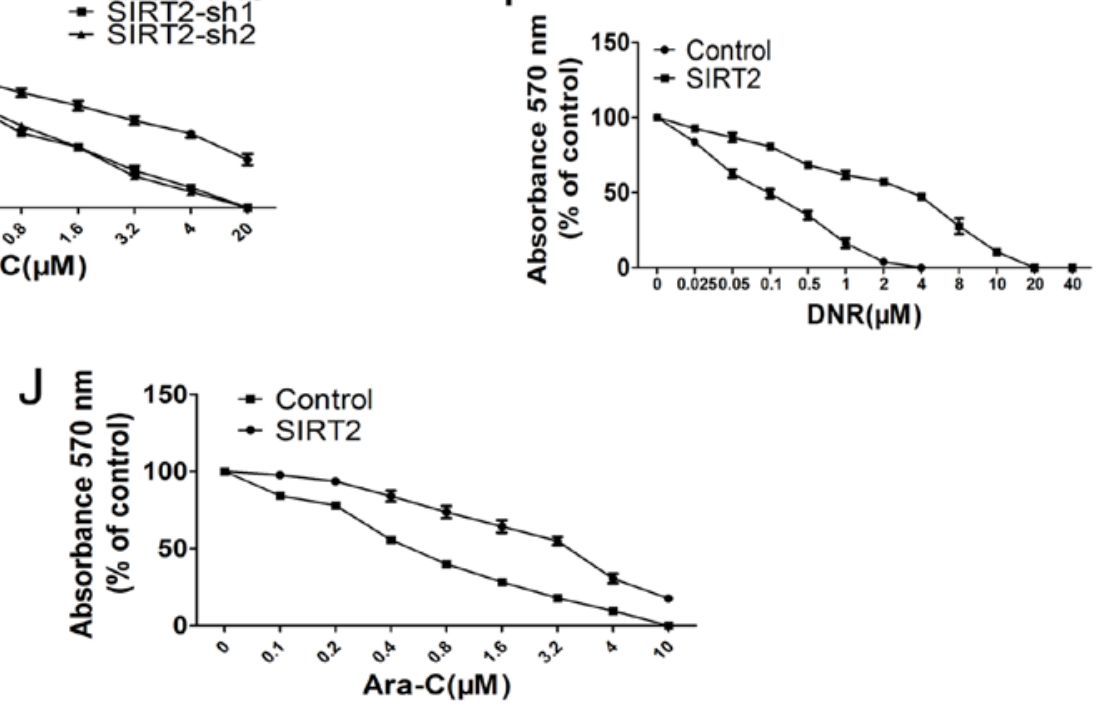

Figure 3. Effects of SIRT2 in HL60/A and HL60 cells on the expression of MRP1 protein, drug accumulation and the sensitivity to drug treatment. (A) The expression of MRP1 protein in SIRT2 silenced HL60/A cells was detected by western blotting. (B) Confocal laser microscope showing the accumulation of doxorubicin in HL60/A cells before and after SIRT2 knockdown. (C and D) The accumulation of doxorubicin in SIRT2 knockdown HL60/A cells and SIRT2 overexpression HL60 cells were detected by FACS analysis. (E) The expression of MRP1 protein in SIRT2 overexpressing HL60 cells. GAPDH was used as an internal control. ${ }^{*} \mathrm{P}<0.05,{ }^{* *} \mathrm{P}<0.01$, compared with the control. (F) Confocal laser microscopy showing the accumulation of doxorubicin in HL60 cells before and after SIRT2 overexpression. (G and H) MTT analysis of HL60/A cells that were, respectively, exposed to different concentrations of daunorubicin (DNR) and arabinocytidine (Ara-C) before and after SIRT2 knockdown. (I and J) MTT analysis of HL60 cells that were, respectively, exposed to different concentrations of daunorubicin (DNR) and arabinocytidine (Ara-C) before and after SIRT2 overexpression. Results are the means of three independent experiments that were determined in triplicate. OD values of untreated control cells were set $100 \%$. All other values refer to the untreated control.

We evaluated the sensitivity of the HL60/A cells to DNR and Ara-C after the SIRT2 was downregulated. The cells were assessed for viability on exposure to DNR and Ara-C, respectively, by using MTT assay and a clear enhancement of sensitivity to DNR and Ara-C was observed in the HL60/A cells when the expression of SIRT2 was downregulated (Fig. 3G and $\mathrm{H}$ ).

In order to detect and quantify apoptosis, we used Annexin V-FITC/PI double staining to determine the effect of SIRT2 knockdown on apoptosis in HL60/A cells with or 
A
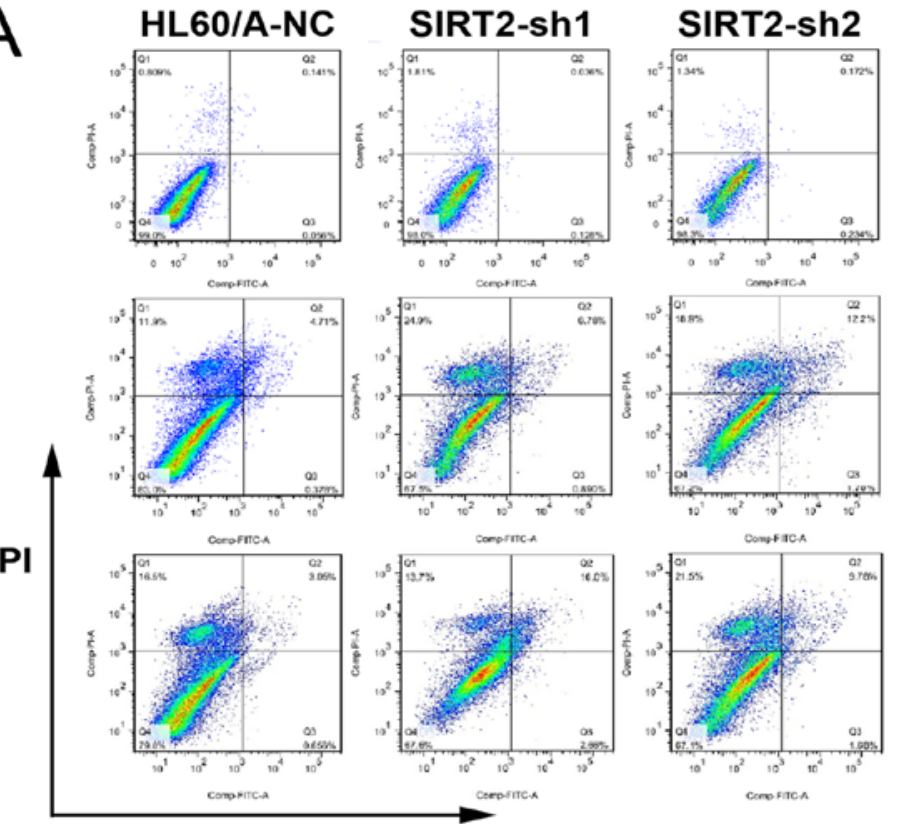

FITC
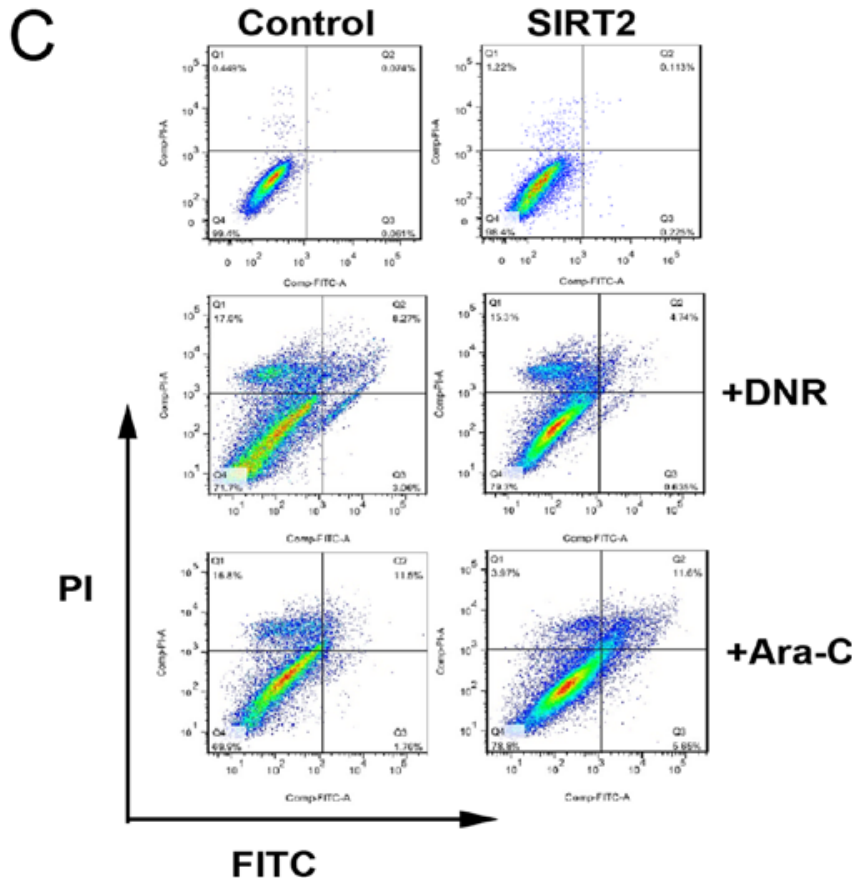
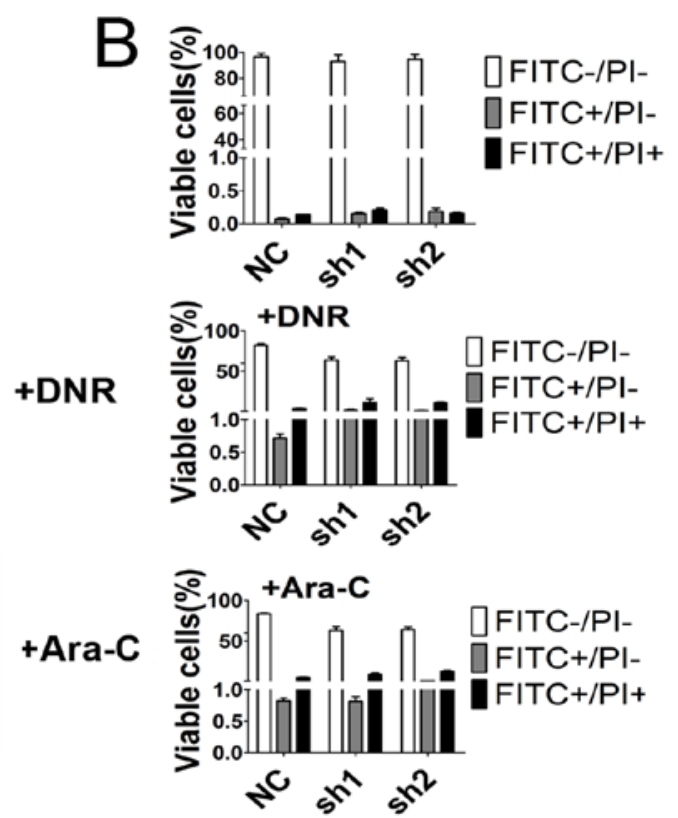

D

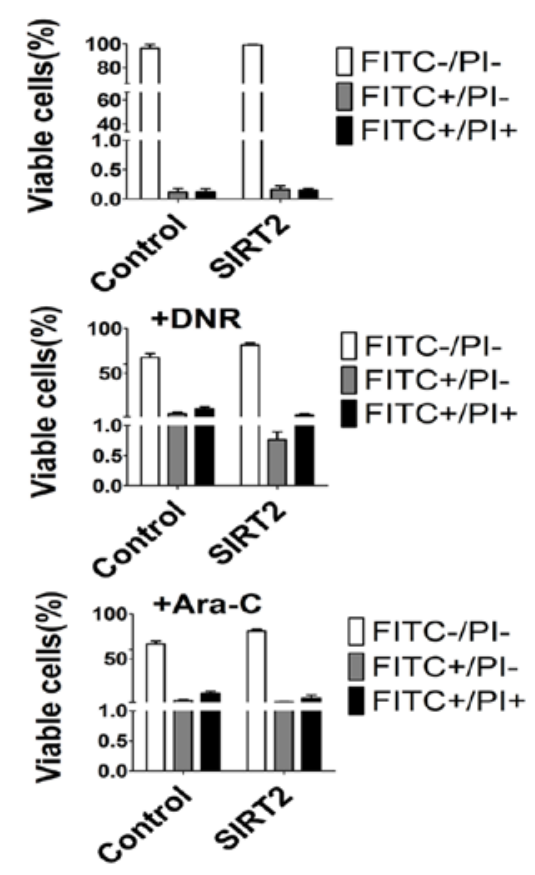

Figure 4. Effects of SIRT2 on drug-induced apoptosis in HL60/A and HL60 cells. (A and B) HL60/A-NC cells and the SIRT2 silenced SIRT2-sh1, SIRT2-sh2 HL60/A cells were treated with or without DNR and Ara-C for $48 \mathrm{~h}$ and subjected to analysis of cell apoptosis by flow cytometric analysis of Annexin V labeling. (C and D) DNR and Ara-C induced apoptosis in HL60 cells and its SIRT2 overexpressing cells were analyzed by flow cytometric analysis of Annexin V labeling.

without DNR or Ara-C treatment. The results showed that SIRT2 downregulation increased slightly the percentage of apoptotic cells, but clearly only after incubation with $0.1 \mu \mathrm{M}$ DNR or $0.5 \mu \mathrm{M}$ Ara-C for $48 \mathrm{~h}$ compared with HL60/A-NC (Fig. 4A and B).

Caspase family proteins have been demonstrated as key mediators of apoptosis, and their activation is taken as a hallmark of apoptosis. We find that the expression of cleaved caspase-3, -7 and -9 and PARP were higher in SIRT2-sh1 and SIRT2-sh2 cells than that in HL60/A-NC after DNR or Ara-C treatment (Fig. 5A). These data indicated that knock- down of SIRT2 enhanced the sensitivity to DNR and Ara-C in HL60/A cells.

SIRT2 overexpression increases MRPI level attenuated drug accumulation and sensitivity to DNR and Ara-C. In order to further study the effect of SIRT2 expression on multidrug resistance, we transfected HL60 cells with SIRT2-overexpression-plasmid and observed an obviously increased expression of SIRT2 in mRNA and protein levels (Fig. 2D and E). The expression of SIRT2 in the HL60 cells after transfection was also detected by a confocal laser microscope 


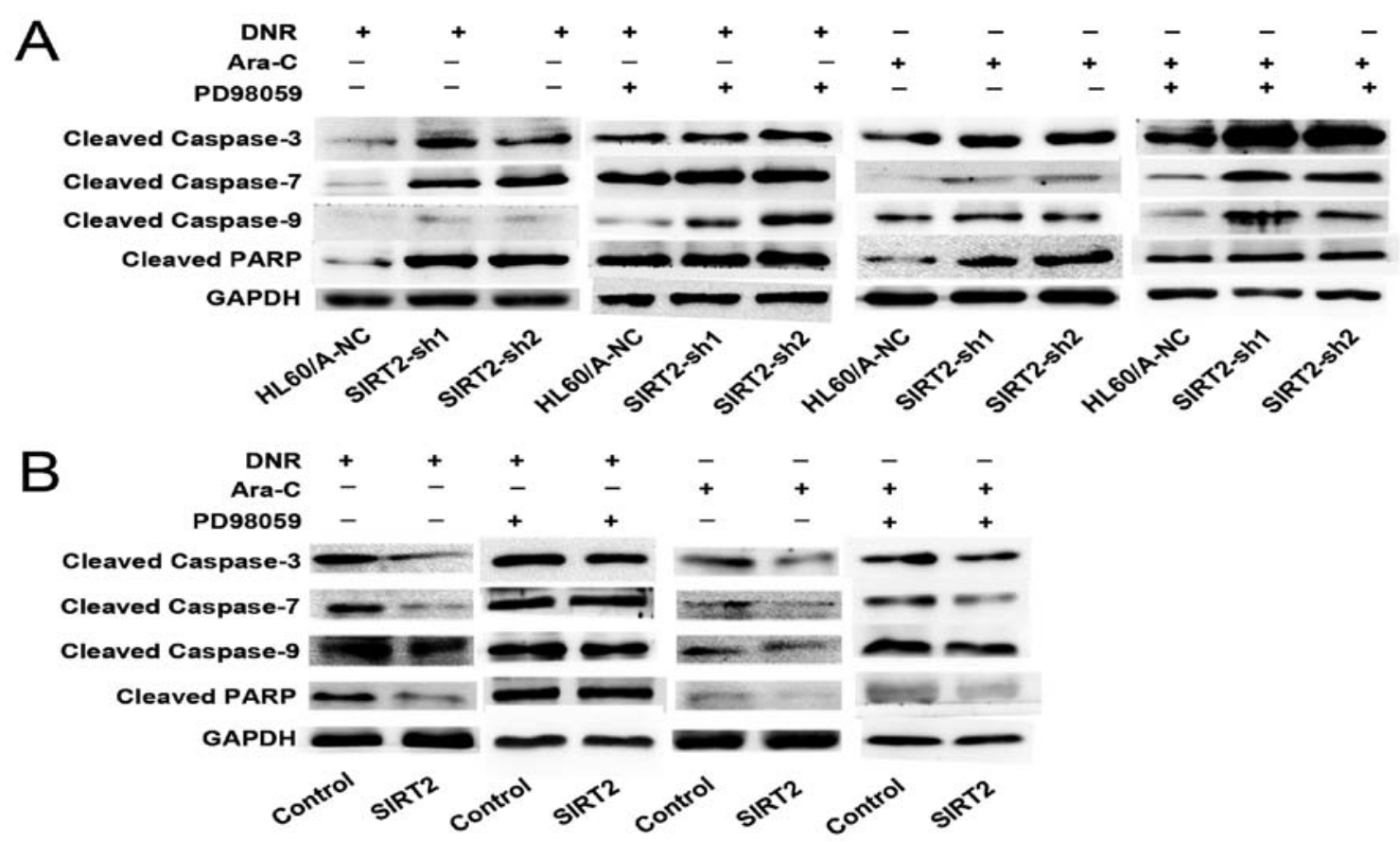

Figure 5. The expression of caspase family proteins in HL60/A and HL60 cells with DNR or Ara-C and with or without PD98059. (A) Cleaved caspase family proteins in SIRT2 silenced HL60/A cells with or without PD98059 after treatment with DNR or Ara-C were evaluated by western blot analyses. (B) The level of cleaved caspase family proteins in SIRT2 overexpressing HL60 cells with or without PD98059 after treatment with DNR or Ara-C. GAPDH was used as an internal control. ${ }^{*} \mathrm{P}<0.05,{ }^{* *} \mathrm{P}<0.01,{ }^{* * *} \mathrm{P}<0.001$, compared with the control.
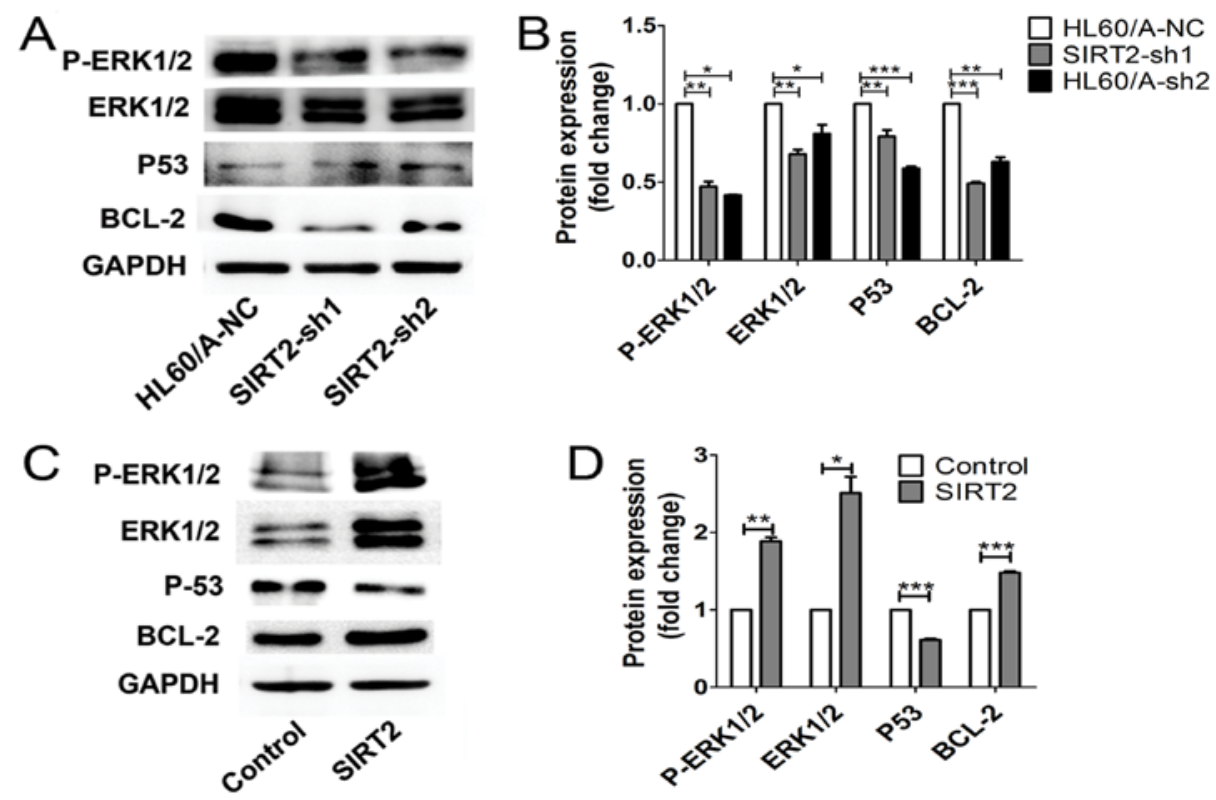

Figure 6. Effects of SIRT2 on the expression of ERK1/2, p53 and BCL-2. (A and B) The expression of P-ERK1/2, ERK1/2, p53 and BCL-2 proteins in SIRT2 silenced HL60/A cells. (C and D) The expression of P-ERK1/2, ERK1/2, p53 and BCL-2 proteins in SIRT2 overexpressed HL60 cells. GAPDH was used as an internal control. ${ }^{*} \mathrm{P}<0.05,{ }^{* *} \mathrm{P}<0.01,{ }^{* * *} \mathrm{P}<0.001$, compared with the control.

(Fig. 2F). The MRP1 expression increased after overexpression of SIRT2 (Fig. 3E).

Confocal laser microscope was used to detect the accumulation of the drug in the cells after SIRT2 overexpression. As expected, after upregulation the expression of SIRT2, in HL60 cells showed decreased uptake of DOX compared with the HL60-control cells (Fig. 3F). We also measured the intracellular fluorescence of DOX by using flow cytometry and obtained similar results (Fig. 3D). The cytotoxicity assay result showed that the sensitivity to DNR and Ara-C was attenuated in the HL60 cells when the expression of SIRT2 was upregulated (Fig. 3I and J). The overexpression of SIRT2, decreased the percentage of apoptotic cells with incubation of DNR and Ara-C (Fig. 4C and D), less cleaved caspase-3, -7 and -9 and PARP protein expression was observed in contrast to HL60-control (Fig. 5B). These results indicated that SIRT2 


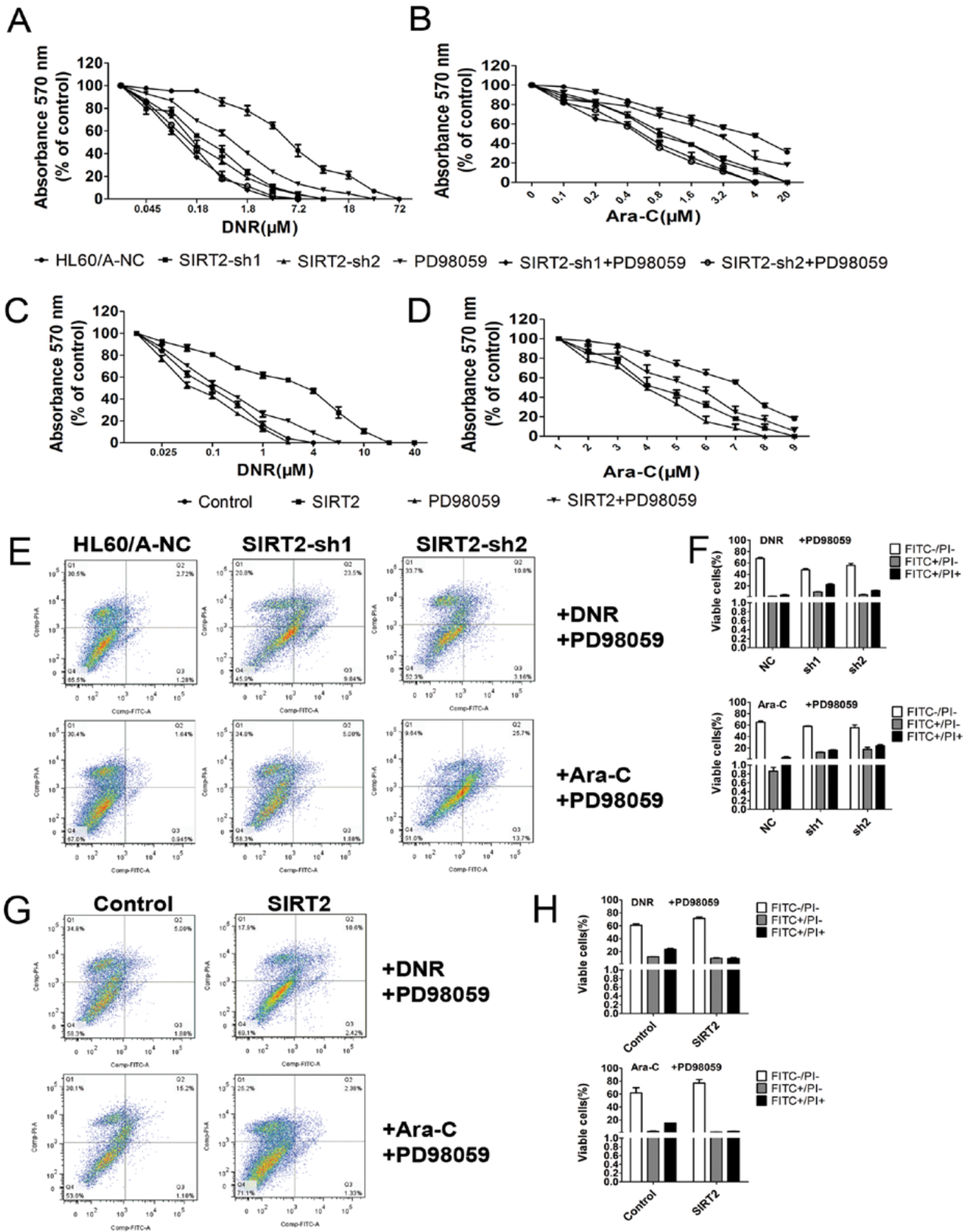

Figure 7. The ERK1/2 signaling pathway is involved in SIRT2-mediated multidrug resistance in AML cell lines. (A and B) MTT analysis of HL60/A cells that were, respectively, exposed to different concentrations of daunorubicin (DNR) and arabinocytidine (Ara-C) before and after SIRT2 knockdown with or without PD98059. (C and D) MTT analysis of HL60 cells that were respectively exposed to different concentrations of DNR and Ara-C before and after SIRT2 overexpression with or without PD98059. Results are the means of three independent experiments that were determined in triplicate. OD values of untreated control cells were set $100 \%$. All other values refer to the untreated control. (E and F) HL60/A-NC cells and the SIRT2 silence HL60/A cells were pre-incubation with PD98059 and treated with DNR and Ara-C for $48 \mathrm{~h}$ and subjected to cell apoptosis analysis by flow cytometric analysis of Annexin V labeling. (G and H) DNR and Ara-C induced apoptosis in HL60 cells and its SIRT2 overexpressing cells which were pre-incubated with PD98059, were analyzed by flow cytometric analysis of Annexin V labeling.

overexpression enhanced the resistance to DNR and Ara-C in HL60 cells, which had a relatively lower expression level of SIRT2, compared to the multidrug resistance HL60/A cells.
The ERK1/2 signaling pathway is involved in SIRT2-mediated multidrug resistance in AML cell lines. We next analyzed the mechanism changing the multidrug resistance effects of the 

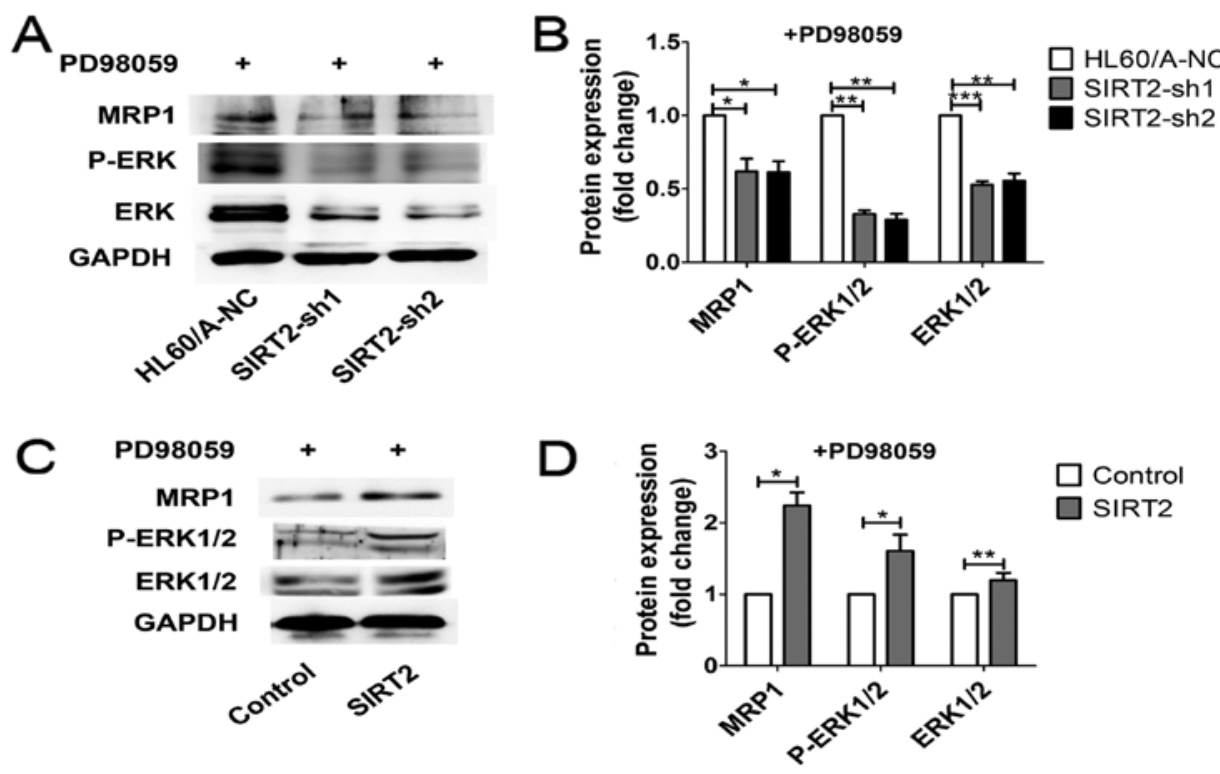

Figure 8. The effect of ERK1/2 pathway on the MRP1 expression. (A and B) The expression of MRP1 protein in SIRT2 silenced HL60/A cells with PD98059 was detected by western blotting. (C and D) The expression of MRP1 protein in SIRT2 overexpressing HL60 cells with PD98059. GAPDH was used as an internal control. ${ }^{*} \mathrm{P}<0.05,{ }^{* *} \mathrm{P}<0.01,{ }^{* * * *} \mathrm{P}<0.001$, compared with the control.

SIRT2 expression in the HL60/A and HL60 cell lines. In our previous study we demonstrated that the MAPKs signaling pathways participate in mediation of the imatinib resistance in CML (21). In the present study, we detected the expression level of ERK1/2, an upstream regulator of p53 as well as BCL-2, an anti-apoptotic protein expressed in most cases of AML and may contribute to drug resistance in $\operatorname{AML}(22,23)$. It has been observed that downregulation of both SIRT1 and SIRT2 can enhance the sensitivity of chemotherapy in mammalian cells via p53-independent mechanism (24), thus, we also evaluated p53. We found that the expression of ERK $1 / 2$ and its phosphorylation as well as BCL-2 were downregulated, while p53 was upregulated in the SIRT2-depleted HL60/A cells (Fig. 6A and B). In contrast, overexpression of the SIRT2 in HL60 cells upregulated ERK1/2 and its phosphorylation and BCL-2, decreased the p53 level (Fig. 6C and D). Thus, these results implied the activation of ERK1/2 signaling might be important to this process.

To further measure the potential influence of manipulating ERK1/2 signaling pathway to this change of multidrug resistance induction exerted by the level of SIRT2, we took advantage of PD98059, a specific MEK1 pharmacological inhibitor, to block its activation and then measured the changes affected by SIRT2 depletion or overexpression. The inhibitor added to the HL60/A cells with SIRT2 depletion did not alone influence the viability of cells on exposure to DNR $(\mathrm{P}<0.05)$ and Ara-C $(\mathrm{P}<0.05)$. Pre-incubation with PD98059 additionally increased the sensitivity of HL60/A (Fig. 7A and B; $\mathrm{P}<0.05)$. The apotosis results show that pre-incubation with PD98059 additionally increased the percentage of apoptotic cells under incubation with DNR and Ara-C (Fig. 7E and F) as well as the caspase family proteins (Fig. 5A). Moreover, the inhibitor was added to the HL60 cells with SIRT2 overexpression, we found that pre-incubation with PD98059 abolished the resistance changes to DNR and Ara-C of HL60 cells (Fig. 7C and $\mathrm{D} ; \mathrm{P}<0.05$ ), reversed the apoptosis changes under treatment with DNR and Ara-C (Fig. 7G and H) and the changes of caspase family proteins, which was caused by SIRT2 overexpressing HL60 cells after treatment with DNR or Ara-C (Fig. 5B). To further elucidate the role of ERK1/2 MAPK signaling in the regulation of MRP1 expression in AML cell lines, we analyzed MRP1 levels in the presence of PD98059 $(50 \mu \mathrm{M})$. MRP1 expression was reduced in HL60/A cells and HL60 cells, additionally decreased in HL60/A cells with SIRT2 depletion and reversed in HL60 cells with SIRT2 overexpression (Fig. 8). According to these results, we suggest that ERK1/2 signaling pathway is involved in these changes of multidrug resistance induction exerted by the level of SIRT2.

\section{Discussion}

Sirtuins, a complex family of proteins (SIRT1-7), are enzymes that require nicotinamide adenine dinucleotide $\left(\mathrm{NAD}^{+}\right)$to catalyze their reactions are present in organisms ranging from bacteria to plants to animals (8-10). Impressive recent progress has been shown with SIRT1, one of the most extensively studied sirtuins. It has been reported to deacetylate the histones $\mathrm{H} 1, \mathrm{H} 3$ and $\mathrm{H} 4$ and regulate transcription factors such as tumor suppressor p53, NF- $\mathrm{kB}$ and Foxo3a (12-14,24-27). Unlike SIRT1, SIRT2 is mostly found in the cytoplasm and deacetylates a number of cytoplasmic proteins, such as $\alpha$-tubulin (28). In addition to this, SIRT2 has also been observed in the nucleus and target histones, NF- $\mathrm{kB}, \mathrm{p} 53$ and FOXO transcription factors $(9,16,17)$. A recent study showed that SIRT2 expression is significantly upregulated in leukemic cells from AML patients and higher in the patients with highrisk AML, which demonstrates the involvement of SIRT2 in the resistance against chemotherapy (29).

In the present study, we not only demonstrated that SIRT2 expression was higher in the AML patients but also higher in other leukemia such as ALL, ET, CML, PV and MPN patients compared to healthy volunteers. As expected, we demon- 
strated that SIRT2 was higher expressed in the relapsed patients suffering from AML than the newly diagnosed AML patients. The results on the expression of SIRT2 in the AML were consistent with the aforementioned report and further demonstrated that SIRT2 participates in the resistance against chemotherapy. To further understand this phenomenon, we used the AML cell line HL60 and its multidrug resistance cell line HL60/A as the cell model to develop the present study. First, we detected the expression of SIRT2 in HL60 and HL60/A cells and the result was consistent with in the patients. Based on this, we constructed two kinds of lentiviral vectors, one for knockdown of SIRT2 in the HL60/A cells and the other for overexpression of SIRT2 in the HL60 cells. We demonstrated that SIRT2-silencing HL60/A cells show more sensitivity to DNR and Ara-C. We observed that more DOX accumulated in SIRT2-silenced HL60/A cells than HL60/A-NC cells. The results we observed in the SIRT2 overexpressing HL60 cells were consistent with the SIRT2silenced HL60/A cells, the SIRT2 overexpression enhanced the drug resistance ability in HL60 cells and more DOX was discharged. All of the data showed that SIRT2 enhanced to multidrug resistance in AML.

To gain more insights into how SIRT2 mediated AML cell multidrug resistance, we measured the activities of multiple signaling pathways after changing the SIRT2 level. We found that SIRT2 knockdown significantly downregulated the phosphorylation of ERK1/2. Overexpression of SIRT2 led to upregulation of the phosphorylation of ERK1/2. To further investigate whether the multidrug resistance of AML cells was mediated by SIRT2 though ERK1/2 pathway, the MEK1 specific inhibitor PD98059 was used. As shown in the results, inhibition of ERK $1 / 2$ by chemical inhibitor indeed assisted the SIRT2 effects. These results implied that SIRT2 mediated the multidrug resistance of AML cells though ERK1/2 signaling pathway.

Furthermore, the expression of p53 and BCL-2 proteins were evaluated. The classical function of $\mathrm{p} 53$ is regulating apoptosis of both the cytosol and mitochondria. These functions involve a direct interaction of p53 with members of the BCL2 family and allowing p53 to function as a so-called BCL2-homology domain-3 (BH3)-only protein (30,31). BCL2 family proteins have a central role in the intrinsic apoptotic pathway. BAX and BAK promote apoptosis by regulating mitochondrial membrane potential, BCL2 and BCL-X1 negatively regulate them, whereas, the BCL2-homology domain-3 (BH3)-only proteins including p53 control these survival proteins (32). Based on the above, a number of p53 inhibitors have been considered as a patron to protect the normal tissues from many of the deleterious side effects of chemotherapies (33). Another report have shown that the balance of $\mathrm{Mdm} 2 / \mathrm{p} 53$ ratio is involved in the sensitization in human breast-, endometrial- and hepatocellular-carcinoma cell lines via EGFR-Raf-MEK-ERK1/2-Mdm2 signaling pathway (34). We hypothesized that ERK1/2-Mdm2-p53-BCL2 may be a pathway related to the multidrug resistance in AML cell lines. However, this hypothesis needs further study.

In conclusion, our results demonstrated that lower expression of SIRT2 declines the multidrug resistance in chemoresistant AML cells. SIRT2 affects the multidrug resistance of AML cell lines via ERK1/2 signaling pathway. These findings shed light on the effect of SIRT2 on the multidrug resistance of AML cells and implied that targeting SIRT2 may yield therapeutic efficacy in the treatment of leukemia diseases.

\section{Acknowledgements}

The present study was supported by grants from the Natural Science Key Program Foundation of Tianjin Technology Commission of China (grant no. 14JCZDJC34900), the National Natural Science General Program Foundation of China (no. 81170510) and the Major Program Foundation of China (no. 81090410).

\section{References}

1. Löwenberg B, Downing JR and Burnett A: Acute myeloid leukemia. N Engl J Med 341: 1051-1062, 1999.

2. Estey E and Döhner H: Acute myeloid leukaemia. Lancet 368: 1894-1907, 2006.

3. DiNardo CD and Cortes JE: New treatment for acute myelogenous leukemia. Expert Opin Pharmacother 16: 95-106, 2015.

4. Vyas P, Appelbaum FR and Craddock C: Allogeneic hematopoietic cell transplantation for acute myeloid leukemia. Biol Blood Marrow Transplant 21: 8-15, 2015.

5. Ross DD: Novel mechanisms of drug resistance in leukemia. Leukemia 14: 467-473, 2000.

6. Legrand O, Zittoun R and Marie JP: Role of MRP1 in multidrug resistance in acute myeloid leukemia. Leukemia 13: 578-584, 1999.

7. Niewiarowski W, Gendaszewska E, Rebowski G, Wójcik M, Mikołajczyk B, Goss W, Soszyński M and Bartosz G: Multidrug resistance-associated protein - reduction of expression in human leukaemia cells by antisense phosphorothioate olignucleotides. Acta Biochim Pol 47: 1183-1188, 2000.

8. Li Z, Xie QR, Chen Z, Lu S and Xia W: Regulation of SIRT2 levels for human non-small cell lung cancer therapy. Lung Cancer 82: 9-15, 2013.

9. Harting K and Knöll B: SIRT2-mediated protein deacetylation: An emerging key regulator in brain physiology and pathology. Eur J Cell Biol 89: 262-269, 2010.

10. Guarente L: Frankin H. Epstein Lecture: Sirtuins, aging, and medicine. N Engl J Med 364: 2235-2244, 2011.

11. Peck B, Chen CY, Ho KK, Di Fruscia P, Myatt SS, Coombes RC, Fuchter MJ, Hsiao CD and Lam EW: SIRT inhibitors induce cell death and p53 acetylation through targeting both SIRT1 and SIRT2. Mol Cancer Ther 9: 844-855, 2010.

12. Audrito V, Vaisitti T, Rossi D, Gottardi D, D'Arena G, Laurenti L, Gaidano G, Malavasi F and Deaglio S: Nicotinamide blocks proliferation and induces apoptosis of chronic lymphocytic leukemia cells through activation of the p53/miR-34a/SIRT1 tumor suppressor network. Cancer Res 71: 4473-4483, 2011.

13. Bradbury CA, Khanim FL, Hayden R, Bunce CM, White DA, Drayson MT, Craddock C and Turner BM: Histone deacetylases in acute myeloid leukaemia show a distinctive pattern of expression that changes selectively in response to deacetylase inhibitors. Leukemia 19: 1751-1759, 2005.

14. Li L, Wang L, Li L, Wang Z, Ho Y, McDonald T, Holyoake TL, Chen $\mathrm{W}$ and Bhatia R: Activation of $\mathrm{p} 53$ by SIRT1 inhibition enhances elimination of CML leukemia stem cells in combination with imatinib. Cancer Cell 21: 266-281, 2012.

15. North BJ and Verdin E: Interphase nucleo-cytoplasmic shuttling and localization of SIRT2 during mitosis. PLoS One 2: e784, 2007.

16. Sunami Y, Araki M, Hironaka Y, Morishita S, Kobayashi M, Liew EL, Edahiro Y, Tsutsui M, Ohsaka A and Komatsu N: Inhibition of the NAD-dependent protein deacetylase SIRT2 induces granulocytic differentiation in human leukemia cells. PLoS One 8: e57633, 2013.

17. Wang F, Nguyen M, Qin FX and Tong Q: SIRT2 deacetylates FOXO3a in response to oxidative stress and caloric restriction. Aging Cell 6: 505-514, 2007. 
18. Hiratsuka M, Inoue $\mathrm{T}$, Toda $\mathrm{T}$, Kimura N, Shirayoshi $\mathrm{Y}$, Kamitani H, Watanabe T, Ohama E, Tahimic CG, Kurimasa A, et al: Proteomics-based identification of differentially expressed genes in human gliomas: Down-regulation of SIRT2 gene. Biochem Biophys Res Commun 309: 558-566, 2003.

19. McGlynn LM, Zino S, MacDonald AI, Curle J, Reilly JE, Mohammed ZM, McMillan DC, Mallon E, Payne AP, Edwards J, et al: SIRT2: Tumour suppressor or tumour promoter in operable breast cancer? Eur J Cancer 50: 290-301, 2014.

20. Chen J, Chan AW, To KF, Chen W, Zhang Z, Ren J, Song C, Cheung YS, Lai PB, Cheng SH, et al: SIRT2 overexpression in hepatocellular carcinoma mediates epithelial to mesenchymal transition by protein kinase B/glycogen synthase kinase-3 $\beta /$ $\beta$-catenin signaling. Hepatology 57: 2287-2298, 2013.

21. Jin W, Li Q, Lin Y, Lu Y, Li H, Wang L, Hu R, Ma L, Wang J and Pang T: Reversal of Imatinib resistance in BCR-ABL-positive leukemia after inhibition of the $\mathrm{Na}^{+} / \mathrm{H}^{+}$exchanger. Cancer Lett 308: 81-90, 2011.

22. Kang MA, Kim MS, Kim JY, Shin YJ, Song JY and Jeong JH: A novel pyrido-thieno-pyrimidine derivative activates p53 through induction of phosphorylation and acetylation in colorectal cancer cells. Int J Oncol 46: 342-350, 2015.

23. Konopleva M, Tari AM, Estrov Z, Harris D, Xie Z, Zhao S, López-Berestein G and Andreeff M: Liposomal Bcl-2 antisense oligonucleotides enhance proliferation, sensitize acute myeloid leukemia to cytosine-arabinoside, and induce apoptosis independent of other antiapoptotic proteins. Blood 95: 3929-3938, 2000.

24. Matsushita N, Takami Y, Kimura M, Tachiiri S, Ishiai M, Nakayama $T$ and Takata M: Role of NAD-dependent deacetylases SIRT1 and SIRT2 in radiation and cisplatin-induced cell death in vertebrate cells. Genes Cells 10: 321-332, 2005.
25. Vaquero A, Scher M, Lee D, Erdjument-Bromage H, Tempst $\mathrm{P}$ and Reinberg D: Human SirT1 interacts with histone H1 and promotes formation of facultative heterochromatin. Mol Cell 16: 93-105, 2004

26. Yeung F, Hoberg JE, Ramsey CS, Keller MD, Jones DR, Frye RA and Mayo MW: Modulation of NF-kappaB-dependent transcription and cell survival by the SIRT1 deacetylase. EMBO J 23: 2369-2380, 2004.

27. Nakagawa T and Guarente L: Sirtuins at a glance. J Cell Sci 124: 833-838, 2011.

28. North BJ and Verdin E: Mitotic regulation of SIRT2 by cyclindependent kinase 1-dependent phosphorylation. J Biol Chem 282: 19546-19555, 2007

29. Dan L, Klimenkova O, Klimiankou M, Klusman JH, van den Heuvel-Eibrink MM, Reinhardt D, Welte K and Skokowa J: The role of sirtuin 2 activation by nicotinamide phosphoribosyltransferase in the aberrant proliferation and survival of myeloid leukemia cells. Haematologica 97: 551-559, 2012

30. Vousden KH and Lane DP: p53 in health and disease. Nat Rev Mol Cell Biol 8: 275-283, 2007.

31. Moll UM, Wolff S, Speidel D and Deppert W: Transcriptionindependent pro-apoptotic functions of p53. Curr Opin Cell Biol 17: 631-636, 2005.

32. Cory S and Adams JM: The Bcl2 family: Regulators of the cellular life-or-death switch. Nat Rev Cancer 2: 647-656, 2002

33. Komarov PG, Komarova EA, Kondratov RV, Christov-Tselkov K, Coon JS, Chernov MV and Gudkov AV: A chemical inhibitor of p53 that protects mice from the side effects of cancer therapy. Science 285: 1733-1737, 1999.

34. Ali I, Damdimopoulou P, Stenius U and Halldin K: Cadmium at nanomolar concentrations activates Raf-MEK-ERK1/2 MAPKs signaling via EGFR in human cancer cell lines. Chem Biol Interact 231: 44-52, 2015 . 